\title{
Bounding reflection length in an affine Coxeter group
}

\author{
Jon McCammond • T. Kyle Petersen
}

Received: 29 November 2010 / Accepted: 6 April 2011 / Published online: 26 April 2011

(C) Springer Science+Business Media, LLC 2011

\begin{abstract}
In any Coxeter group, the conjugates of elements in the standard minimal generating set are called reflections, and the minimal number of reflections needed to factor a particular element is called its reflection length. In this article we prove that the reflection length function on an affine Coxeter group has a uniform upper bound. More precisely, we prove that the reflection length function on an affine Coxeter group that naturally acts faithfully and cocompactly on $\mathbb{R}^{n}$ is bounded above by $2 n$, and we also show that this bound is optimal. Conjecturally, spherical and affine Coxeter groups are the only Coxeter groups with a uniform bound on reflection length.
\end{abstract}

Keywords Coxeter group $\cdot$ Reflection length

Every Coxeter group $W$ has two natural generating sets: the set $S$ used in its standard presentation and the set $R$ of reflections formed by collecting all conjugates of the elements in $S$. The first generating set leads to the standard length function $\ell_{S}: W \rightarrow \mathbb{N}$, and the second is used to define the reflection length function $\ell_{R}: W \rightarrow \mathbb{N}$. When $W$ is finite, both length functions are uniformly bounded for trivial reasons and are fairly well understood. ${ }^{1}$ On the other hand, when $S$ is infinite, i.e., when $W$ has infinite rank,

\footnotetext{
${ }^{1}$ For finite $W$, these generating sets and length functions exhibit an interesting duality: the maximum value of $\ell_{S}$ is $|R|$, and the maximum value of $\ell_{R}$ is $|S|$. See [1] for further details and for additional illustrations of this phenomenon.
}

Work of McCammond partially supported by an NSF grant.

Work of Petersen partially supported by an NSA Young Investigator grant.

J. McCammond

Mathematics Department, University of California, Santa Barbara, Santa Barbara, CA 93106, USA

T.K. Petersen $(\varangle)$

Department of Mathematical Sciences, DePaul University, 2320 N. Kenmore, Chicago, IL 60614, USA

e-mail: tpeter21@depaul.edu 
it is easy to show that both length functions are unbounded by considering products of elements in $S$. Thus, we restrict our attention to infinite Coxeter groups of finite rank. For these Coxeter groups, the function $\ell_{S}$ is always unbounded because there are only finitely many group elements of a given length as a consequence of the fact that $S$ is finite. Our main result is that $\ell_{R}$ remains bounded for affine Coxeter groups, and we provide an explicit optimal upper bound.

Theorem A (Explicit affine upper bounds) If $W$ is an affine Coxeter group that naturally acts faithfully and cocompactly on $\mathbb{R}^{n}$, then every element of $W$ has reflection length at most $2 n$, and there exist elements in $W$ with reflection length equal to $2 n$.

The article is structured as follows. The first two sections recall basic facts, the third establishes a key technical result, and the fourth contains the proof of our main result. The final section contains a conjecture about infinite nonaffine Coxeter groups.

\section{Reflection length}

We assume that the reader is familiar with the basic theory of reflection groups (as described, for example, in [4]), and we generally follow the standard notational conventions.

Definition 1.1 (Reflection length) Let $W$ be a Coxeter group with standard generating set $S$. A reflection in $W$ is any conjugate of an element of $S$, and we use $R$ to denote the set of all reflections in $W$. In other words, $R=\left\{w s w^{-1} \mid s \in S, w \in W\right\}$. We should note that unless $W$ is a finite group, $R$ is an infinite set. For any element $w \in W$, its reflection length $\ell_{R}(w)$ is the minimal number of reflections whose product is $w$. Thus $w=r_{1} r_{2} \cdots r_{k}$ with $r_{i} \in R$ means $\ell_{R}(w) \leq k$. Alternatively, $\ell_{R}(w)$ can be defined as the combinatorial distance from the vertex labeled by the identity to the vertex labeled by $w$ in the Cayley graph of $W$ with respect to $R$.

Since combinatorial distance defines a metric on the vertex set of any graph and Cayley graphs are homogeneous in the sense that there is a vertex-transitive group action, metric properties of the distance function translate into properties of $\ell_{R}$. Symmetry and the triangle inequality, for example, imply that $\ell_{R}(w)=\ell_{R}\left(w^{-1}\right)$, and $\ell_{R}(u v) \leq \ell_{R}(u)+\ell_{R}(v)$, respectively.

It is sufficient to investigate reflection length in irreducible Coxeter groups because of the following elementary result.

Proposition 1.2 (Reducible Coxeter groups) When $W$ is a reducible Coxeter group, its standard generating set $S$ has a nontrivial partition $S=S_{1} \sqcup S_{2}$ in which every element in $S_{1}$ commutes with every element in $S_{2}$. In this context, $W=W_{1} \times W_{2}$ where $W_{i}$ denotes the parabolic subgroup generated by $S_{i}$, the reflections $R$ in $W$ can be partitioned as $R=R_{1} \sqcup R_{2}$ where $R_{i}$ denotes the reflections in $W_{i}$, and when $w \in W$ is written in the form $w=w_{1} w_{2}$ with $w_{i} \in W_{i}$, we have $\ell_{R}(w)=\ell_{R_{1}}\left(w_{1}\right)+$ $\ell_{R_{2}}\left(w_{2}\right)$. 


\section{Affine Coxeter groups}

Next we review the construction of an affine Coxeter group from a crystallographic root system.

Definition 2.1 (Affine Coxeter groups) Recall that a crystallographic root system $\Phi$ is a finite collection of vectors that span a real Euclidean space $V$ satisfying a few elementary properties, the main ones being that $\Phi$ contains at most two vectors from each line through the origin, $\Phi$ is stable under the reflections defined by these vectors, and an integrality condition on their inner products. See [4] for a precise definition. An affine Coxeter group $W$ can be constructed from $\Phi$ as follows. For each $\alpha \in \Phi$ and $i \in \mathbb{Z}$, let $H_{\alpha, i}$ denote the (affine) hyperplane of solutions to the equation $\langle x, \alpha\rangle=i$ where the brackets denote the standard inner product on $V$. The unique nontrivial isometry of $V$ that fixes $H_{\alpha, i}$ pointwise is a reflection that we call $r_{\alpha, i}$. The collection $R=\left\{r_{\alpha, i} \mid \alpha \in \Phi, i \in \mathbb{Z}\right\}$ generates the affine Coxeter group $W$, and $R$ is its set of reflections in the sense of Definition 1.1. A standard minimal generating set $S$ can be obtained by restricting to those reflections that reflect across the facets of a certain polytope in $V$.

Remark 2.2 (No finite factors) Every irreducible affine Coxeter group can be constructed from its crystallographic root system as described in Definition 2.1, but the construction also works equally well when the root system is reducible. It is not, however, sufficient to construct arbitrary reducible affine Coxeter groups because it always constructs affine Coxeter groups with affine irreducible components (i.e., no finite irreducible components). The affine Coxeter groups constructible from a root system in this way can also be characterized as those that naturally act faithfully and cocompactly on some Euclidean space.

For each affine Coxeter group $W$ constructed from a root system $\Phi$, there is a finite Coxeter group $W_{0}$ related to $W$ in two distinct ways.

Definition 2.3 (Subgroups and quotients) We abbreviate $r_{\alpha, 0}$ and $H_{\alpha, 0}$ as $r_{\alpha}$ and $H_{\alpha}$, respectively. The hyperplanes $H_{\alpha}$ are precisely the ones that contain the origin, and the reflections $R_{0}=\left\{r_{\alpha} \mid \alpha \in \Phi\right\}$ generate a finite Coxeter group $W_{0}$ that contains all elements of $W$ that fix the origin. This embeds $W_{0}$ as a subgroup of $W$. There is a group homomorphism $p: W \rightarrow W_{0}$ defined by sending each generating reflection $r_{\alpha, i}$ in $W$ to $r_{\alpha}$ in $W_{0}$.

Because the map $p: W \rightarrow W_{0}$ sends $R$ to $R_{0}$, it sends reflection factorizations to reflection factorizations, proving the following.

Proposition 2.4 (Lengths and quotients) If the map $p: W \rightarrow W_{0}$ sends $u$ to $u_{0}$, then $\ell_{R}(u) \geq \ell_{R_{0}}\left(u_{0}\right)$.

Finite Coxeter groups such as $W_{0}$ are also known as spherical Coxeter groups because they act by isometries on spheres, and we digress for a moment to recall a 
few of their key properties. In a finite Coxeter group, reflection length has a geometric interpretation that yields a spherical version of Theorem A as an immediate corollary. The following properties were observed by Carter in [2].

Proposition 2.5 (Spherical reflection length) The reflection length of an element $w$ in a finite Coxeter group $W_{0}$ is equal to the codimension of the subspace of vectors that $w$ fixes in the standard orthogonal representation of $W_{0}$. In addition, a reflection factorization $w=r_{1} r_{2} \cdots r_{m}$ is of minimum length if and only if the vectors normal to the hyperplanes of these reflections are linearly independent.

Corollary 2.6 (Spherical upper bounds) Let $W_{0}$ be a finite Coxeter group of rank $n$, i.e., one whose standard representation acts on $\mathbb{R}^{n}$ by orthogonal transformations. For all $w \in W_{0}, \ell_{R}(w) \leq n$, and for elements that only fix the origin, $\ell_{R}(w)=n$. More concretely, multiplying the elements in its standard minimal generating set $S_{0}$ produces an element $w$ with $\ell_{R}(w)=n$.

Proposition 2.5 also has consequences for some elements in $W$.

Corollary 2.7 (Linearly independent roots) If $w=r_{1} r_{2} \cdots r_{m}$ is a reflection factorization of $w \in W$ in which the roots of the reflections are linearly independent, then $\ell_{R}(w)=m$.

Proof The factorization shows that $\ell_{R}(w) \leq m$. For the lower bound, note that each reflection $r_{i} \in R$ is $r_{\alpha_{i}, c_{i}}$ for some $\alpha_{i} \in \Phi$ and $c_{i} \in \mathbb{Z}$, and by hypothesis the roots $\alpha_{i}$ are linearly independent. If we let $w_{0}=p(w)$, then by Propositions 2.4 and 2.5 we have $\ell_{R}(w) \geq \ell_{R_{0}}\left(w_{0}\right)=m$.

Although we never use the following result established by Solomon in [6], we mention it because it highlights how reflection length captures fundamental aspects of the behavior of finite Coxeter groups.

Proposition 2.8 (Solomon's factorization formula) For each finite Coxeter group $W_{0}$, the polynomial recording the distribution of reflection lengths factors completely over the integers. In particular,

$$
f(x)=\sum_{w \in W_{0}} x^{\ell_{R}(w)}=\prod_{i=1}^{n}\left(1+e_{i} x\right),
$$

where the $e_{i}$ are the exponents of $W_{0}$.

We now return to the structure of the affine Coxeter group $W$ constructed from a root system $\Phi$.

Definition 2.9 (Coroots) For each root $\alpha \in \Phi$, there is a corresponding $\operatorname{coroot} \alpha^{\vee}=$ $c \alpha$ with $c=\frac{2}{\langle\alpha, \alpha\rangle}$. The collection of all coroots is denoted $\Phi^{\vee}$, and the integral linear combinations of vectors in $\Phi^{\vee}$ are a lattice $L=\mathbb{Z} \Phi^{\vee} \cong \mathbb{Z}^{n}$ called the coroot lattice. 
The isomorphism with $\mathbb{Z}^{n}$ is a result of the existence of a subset $\Delta^{\vee} \subset \Phi^{\vee}$ of linearly independent vectors that form a basis for $L$.

The coroot lattice describes the translations in $W$.

Definition 2.10 (Translations) A translation is a map that shifts each point by the same vector $\lambda$, and we let $t_{\lambda}$ denote the map sending each point $x \in V$ to $x+\lambda$. For each $\alpha \in \Phi$, consider the product $r_{\alpha, 1} r_{\alpha}$. Reflecting through parallel hyperplanes produces a translation in the $\alpha$ direction, and the exact translation is $t_{\alpha^{\vee}}$. Because $t_{\mu} t_{\nu}=t_{\mu+\nu}$, there is a translation of the form $t_{\lambda}$ in $W$ for each $\lambda \in L$, and the set $T=$ $\left\{t_{\lambda} \mid \lambda \in L\right\}$ forms an abelian subgroup of $W$. In fact, these are the only translations that are contained in $W$.

The subgroup $T$ is also the kernel of the map $p: W \rightarrow W_{0}$, and $W$ can be viewed as a semidirect product $W=W_{0} \ltimes T$.

Proposition 2.11 (Normal forms) For each element $w \in W$, there is a unique factorization $w=t_{\lambda} w_{0}$ where $t_{\lambda}$ is a translation with $\lambda \in L$ and $w_{0}$ is an element in $W_{0}$.

Proof If such an expression exists, then $w_{0}$ is the image of $w$ under the map $p: W \rightarrow$ $W_{0}$, and $\lambda$ is the image of the origin under $w$ (keeping in mind that elements of $W$ act on $V$ in function notation so that composition is from right to left). This proves uniqueness. For existence, define $w_{0}=p(w) \in W_{0} \subset W$ and consider the element $w w_{0}^{-1}$. Since it is in the kernel of $p$, it is translation in the form $t_{\lambda}$ for some $\lambda \in L$.

\section{Translation dimension}

In this section we introduce the notion of the dimension of a vector in the coroot lattice. As above, $W$ is an affine Coxeter group, constructed from a root system $\Phi$, acting on a vector space $V$.

Definition 3.1 (Real dimension) We call a subspace of $V$ spanned by a collection of coroots in $\Phi^{\vee}$ a real coroot subspace, and we say that a vector $\lambda$ has real dimension $k$ when $\lambda$ is contained in a $k$-dimensional coroot subspace but is not contained in any real coroot subspace of strictly smaller dimension. Since it is always possible to find a coroot basis for each coroot subspace, real dimension $k$ means that $\lambda=$ $c_{1} \alpha_{1}^{\vee}+c_{2} \alpha_{2}^{\vee}+\cdots+c_{k} \alpha_{k}^{\vee}$ for some $c_{i} \in \mathbb{R}$ and $\alpha_{i}^{\vee} \in \Phi^{\vee}$ but that no such expression exists with fewer summands.

Remark 3.2 (Nonunique subspaces) There may be more than one $k$-dimensional coroot subspace containing a vector $\lambda \in L$ of real dimension $k$ because coroot subspaces are not closed under intersection. Consider the $D_{n}$ root system $\Phi=\left\{ \pm e_{i} \pm e_{j} \mid 1 \leq\right.$ $i<j \leq n\}$. Every root has norm 2, so $\alpha=\alpha^{\vee}$ and $\Phi=\Phi^{\vee}$. The translation $t_{\lambda}$ with $\lambda=2 e_{1} \in L$ is two-dimensional because $2 e_{1}$ is in the span of $\left\{e_{1}+e_{j}, e_{1}-e_{j}\right\}$ for any $j>1$ but as $j$ varies we get distinct 2-planes containing $\lambda$. They intersect along the $e_{1}$-axis, but this line is not a real coroot subspace. 
The following definition is very similar.

Definition 3.3 (Integral dimension) We say a vector $\lambda \in L$ has integral dimension $k$ when $\lambda$ can be expressed in the form $\lambda=c_{1} \alpha_{1}^{\vee}+c_{2} \alpha_{2}^{\vee}+\cdots+c_{k} \alpha_{k}^{\vee}$ for some $c_{i} \in \mathbb{Z}$ and $\alpha_{i}^{\vee} \in \Phi^{\vee}$ but no such expression exists with fewer summands. The restriction to integral coefficients means that only vectors in $L$ have a dimension in this sense. When $\lambda$ has integral dimension $k$, we say $\lambda$ is $k$-dimensional as is the corresponding translation $t_{\lambda}$. Note that the real dimension of $\lambda$ is a lower bound on its integral dimension.

Proposition 3.4 (Dimensions) If $W$ is an affine Coxeter group constructed from a root system $\Phi$ and naturally acts faithfully and cocompactly on $V=\mathbb{R}^{n}$, then every vector in its coroot lattice has integral dimension at most $n$, and $n$-dimensional vectors do exist.

Proof The first assertion is a consequence of the fact that $L \cong \mathbb{Z}^{n}$ is a lattice with a $\mathbb{Z}$-basis in $\Phi^{\vee}$. For the second assertion, note that any vector $\lambda \in L$ that does not lie in the union of the finite number of proper subspaces through the origin that are spanned by coroots has real dimension $n$ and thus integral dimension at least $n$.

The integral dimension of a vector $\lambda \in L$ bounds how hard it is to move the origin to $\lambda$ using reflections. To prove this assertion, we need an elementary result about factorizations.

Lemma 3.5 (Rewriting factorizations) Let $W$ be a Coxeter group with reflections $R$, and let $w=r_{1} r_{2} \cdots r_{m}$ be a reflection factorization. For any selection $1 \leq i_{1}<i_{2}<$ $\cdots<i_{k} \leq m$ of positions, there is a length $m$ reflection factorization of $w$ whose first $k$ reflections are $r_{i_{1}} r_{i_{2}} \cdots r_{i_{k}}$ and another length $m$ reflection factorization of $w$ where these are the last $k$ reflections in the factorization.

Proof Because reflections are closed under conjugation, for any reflections $r$ and $r^{\prime}$, there exist reflections $r^{\prime \prime}$ and $r^{\prime \prime \prime}$ such that $r r^{\prime}=r^{\prime \prime} r$ and $r^{\prime} r=r r^{\prime \prime \prime}$. Iterating these rewriting operations allows us to move the selected reflections into the desired positions without altering the length of the factorization.

In preparation for the next result recall that $t_{\alpha^{\vee}}=r_{\alpha, 1} r_{\alpha}$ and note that because the hyperplanes $H_{\alpha, i}$ are evenly spaced, the product $r_{\alpha, i+1} r_{\alpha, i}$ is also $t_{\alpha}$ for every $i \in \mathbb{Z}$. More generally, $r_{\alpha, i+j} r_{\alpha, i}=t_{j \alpha^{\vee}}$.

Proposition 3.6 (Moving points) If $\lambda \in L$ has integral dimension $k$, then $\ell_{R}\left(t_{\lambda}\right) \leq$ $2 k$, and there is an element $u \in W$ with $\ell_{R}(u) \leq k$ that sends the origin to $\lambda$.

Proof By the definition of integral dimension there is an equation of the form $\lambda=$ $c_{1} \alpha_{1}^{\vee}+c_{2} \alpha_{2}^{\vee}+\cdots+c_{k} \alpha_{k}^{\vee}$, and the formulas $t_{\mu+v}=t_{\mu} t_{v}$ and $t_{j \alpha^{\vee}}=r_{\alpha, j} r_{\alpha}$ show that $t_{\lambda}$ has a length $2 k$ reflection factorization of the form $t_{\lambda}=\left(r_{\alpha_{1}, c_{1}} r_{\alpha_{1}}\right)\left(r_{\alpha_{2}, c_{2}} r_{\alpha_{2}}\right) \ldots$ $\left(r_{\alpha_{k}, c_{k}} r_{\alpha_{k}}\right)$. Next, by Lemma 3.5 there is another length $2 k$ reflection factorization 
of $t_{\lambda}$ where the final $k$ reflections are $r_{\alpha_{1}} r_{\alpha_{2}} \cdots r_{\alpha_{k}}$. Since all of these reflections fix the origin, the product of the first $k$ reflections is an element $u$ that sends the origin to $\lambda$.

Note that just as there can be distinct minimal expressions for $\lambda$ (Remark 3.2), there are often distinct elements of reflection length $k$ that send the origin to $\lambda$.

Theorem 3.7 (Equivalent definitions) For each $\lambda \in L$, the real dimension of $\lambda$, the integral dimension of $\lambda$, and the minimal reflection length of an element sending the origin to $\lambda$ are equal.

Proof Let $k_{r}, k_{d}$, and $k_{m}$ be three numbers at issue in the order listed. Proposition 3.6 shows that $k_{d} \geq k_{m}$. Next, let $u$ be an element sending the origin to $\lambda$. Because a reflection $r_{\alpha, i}$ moves points in the $\alpha^{\vee}$ direction, $\lambda$ is in the span of the coroots associated to the reflections in a minimal length reflection factorization of $u$. Thus $k_{m} \geq k_{r}$. And finally, let $V^{\prime}$ be a $k_{r}$-dimensional coroot subspace of $V$ containing $\lambda$. The set $\Phi^{\prime}=\Phi \cap V^{\prime}$ satisfies the requirements to be a root system, and $L^{\prime}=L \cap V^{\prime}$ is its coroot lattice. ${ }^{1}$ Because $\lambda$ lies in the lattice $L^{\prime}, k_{r} \geq k_{d}$. The combination $k_{d} \geq k_{m} \geq k_{r} \geq k_{d}$ shows that all three are equal.

\section{Bounding reflection length}

We are now ready to prove our main result.

Proposition 4.1 (Bounds) Let $W$ be an affine Coxeter group naturally acting on $\mathbb{R}^{n}$ (so that $n$ is the rank of $W_{0}$ ). If $w \in W$ has the form $w=t_{\lambda} w_{0}$ where $t_{\lambda}$ is a $k$-dimensional translation and $w_{0} \in W_{0}$ is an element fixing the origin, then $k \leq$ $\ell_{R}(w) \leq k+n$. In particular, every element $w \in W$ has $\ell_{R}(w) \leq 2 n$.

Proof The lower bound, $\ell_{R}(w) \geq k$, follows from Theorem 3.7. By Proposition 3.6 and Theorem 3.7 there is an element $u$ with $\ell_{R}(u)=k$ that sends the origin to $\lambda$. Because the element $v_{0}=u^{-1} w$ fixes the origin, it is in $W_{0}$, and $\ell_{R}\left(v_{0}\right) \leq n$ by Corollary 2.6. Thus $\ell_{R}(w)=\ell_{R}\left(u v_{0}\right) \leq \ell_{R}(u)+\ell_{R}\left(v_{0}\right) \leq k+n$. For the final assertion, note that every element can be written in this form (Proposition 2.11) and every translation is $k$-dimensional for some $k \leq n$ (Proposition 3.4).

Based on the proof of this proposition, one might conjecture that each $w=t_{\lambda} w_{0}$ with $k$-dimensional $\lambda$ has another factorization $w=u v_{0}$ with $v_{0} \in W_{0}, \ell_{R}\left(v_{0}\right)=$ $\ell_{R}(p(w))$ and $\ell_{R}(w)=\ell_{R}(u)+\ell_{R}\left(v_{0}\right)$. This is not always the case.

\footnotetext{
${ }^{1}$ This fact is well known, but it is slightly subtle in that it can fail when $V^{\prime}$ is not a coroot subspace. The argument goes as follows. The pointwise stabilizer of the orthogonal complement of $V$ is a parabolic subgroup with root system $\Phi^{\prime}=\Phi \cap V$, and since $\Phi^{\prime}$ is a parabolic subsystem of $\Phi$, one may choose a simple system of roots for $\Phi$ which contains one for $\Phi^{\prime}$. Because $V^{\prime}$ is a coroot subspace, it is linearly spanned by $\Phi^{\prime}$ and has this simple system of $\Phi^{\prime}$ as a basis. At this point the equality $L^{\prime}=L \cap V^{\prime}$ becomes clear.
} 
Example 4.2 (Exact bounds) Let $\alpha_{i j}$ denote the vector $e_{i}-e_{j}$, let $\Phi=\left\{\alpha_{i j} \mid 1 \leq\right.$ $i, j \leq 4\}$ be the $A_{3}$ root system with coroot lattice $L$, and consider the elements $w_{i j k}=r_{\alpha_{12}, i} r_{\alpha_{23}, j} r_{\alpha_{34}, k}$. By Corollary $2.7 \ell_{R}\left(w_{i j k}\right)=3$. All of these elements are sent under $p: W \rightarrow W_{0}$ to $w_{000}=r_{\alpha_{12}} r_{\alpha_{23}} r_{\alpha_{34}}$, which cyclically permutes the four coordinates moving the point $(x, y, z, w)$ to $(w, x, y, z)$. The minimum length reflection factorizations of $w_{000}$ in $W_{0}$ are well known, and they are encoded by maximal chains in the lattice $\mathrm{NC}_{4}$ of noncrossing partitions on four elements [5]. Every reflection in $R_{0}$ occurs in some factorization of $w_{000}$, but there exists a pair of reflections, $r_{\alpha_{13}}$ and $r_{\alpha_{24}}$, representing a "crossing" partition that cannot both occur in the same factorization. By varying $i, j$, and $k$, the elements $w_{i j k}$ produce all elements of the form $t_{\lambda} w_{000}$ with $\lambda \in L$. Thus, for a careful choice of values, the vector $\lambda$ is in the span of $\alpha_{13}$ and $\alpha_{24}$, and this is the unique minimal dimensional coroot subspace containing $\lambda$. If $u$ is a product of two reflections sending such a $\lambda$ to the origin, then the coroots involved in this product must be $\alpha_{13}$ and $\alpha_{24}$. As a consequence, there is no length 3 reflection factorization of the form $w_{i j k}=u v_{0}$ since the projection of such a factorization to $W_{0}$ would be a length 3 reflection factorization of $w_{000}$ containing both $r_{\alpha_{13}}$ and $r_{\alpha_{24}}$, which is known not to exist.

Proposition 4.3 (Optimality) If $W$ is a affine Coxeter group and $w=t_{\lambda}$ is a $k$ dimensional translation, then $\ell_{R}(w)=2 k$. In particular, $n$-dimensional translations have reflection length $2 n$.

Proof Being a translation in $W, w=t_{\lambda}$ for some $\lambda \in L$ and by definition of dimension $\lambda=c_{1} \alpha_{1}^{\vee}+c_{2} \alpha_{2}^{\vee}+\cdots+c_{k} \alpha_{k}^{\vee}$ for $\alpha_{i}^{\vee} \in \Phi^{\vee}$ and $c_{i} \in \mathbb{Z}$. By Proposition 3.6, $\ell_{R}(w) \leq 2 k$. To show that $2 k$ is also a lower bound, suppose that $w=r_{1} r_{2} \cdots r_{m}$ with each $r_{i}=r_{\alpha_{i}, c_{i}} \in R$. Because the reflection $r_{i}$ moves points in the $\alpha_{i}^{\vee}$ direction and $\lambda$ has real dimension $k$ (Theorem 3.7), the coroots $\alpha_{i}^{\vee}$ must span a subspace of dimension at least $k$. Next, we may assume that the first $k$ reflections have linearly independent coroots since it is possible to use Lemma 3.5 to move any $k$ reflections with linearly independent coroots to the front. This alters the reflection factorization of $w$ but leaves the total length unchanged. Let $u=r_{1} r_{2} \cdots r_{k}$ and $v=r_{k+1} \cdots r_{m}$ so that $w=u v$, let $u_{0}=p(u)$ and $v_{0}=p(v)$ where $p$ is the homomorphism $p: W \rightarrow W_{0}$, and note that $\ell_{R_{0}}\left(u_{0}\right)=k$ by Proposition 2.5. Since $w$ is a translation, $p(w)$ is the identity, and $v_{0}=u_{0}^{-1}$. Finally, by Proposition 2.4 we have $\ell_{R}(v) \geq \ell_{R_{0}}\left(v_{0}\right)=\ell_{R_{0}}\left(u_{0}^{-1}\right)=\ell_{R_{0}}\left(u_{0}\right)=k$, which means that any reflection factorization of $v$ has length at least $k$. This implies that $m \geq 2 k$, and as a consequence every reflection factorization of $w$ has length at least $2 k$.

Theorem B (Explicit affine upper bounds) If $W$ is an affine Coxeter group that naturally acts faithfully and cocompactly on $\mathbb{R}^{n}$, then every element of $W$ has reflection length at most $2 n$, and there exist elements in $W$ with reflection length equal to $2 n$.

Proof Proposition 4.1 shows that $2 n$ is an upper bound. Propositions 3.4 and 4.3 show that it is optimal.

Remark 4.4 (Finite factors) The cocompactness assumption in Theorem A essentially means that $W$ does not have any finite irreducible factors (Remark 2.2). When finite 
irreducible factors are present, the optimal upper bound can be lowered accordingly. In particular, if $W=W_{f} \times W_{a}$, where $W_{f}$ is the product of the finite irreducible factors, and $W_{a}$ is the product of the affine irreducible factors, and $\mathbb{R}^{n}=\mathbb{R}^{n_{f}} \oplus \mathbb{R}^{n_{a}}$ is the orthogonal decomposition preserved by this splitting, then by Proposition 1.2, Corollary 2.6, and Theorem A the optimal upper bound for the reflection length function on $W$ is $n_{f}+2 n_{a}=2 n-n_{f}$.

We should note that we have not found an elementary way to compute $\ell_{R}(w)$ exactly for a generic $w$ in an affine Coxeter group $W$. Techniques for easily computing $\ell_{R}(w)$ would be useful. For example, they would enable one to investigate whether the polynomial $f_{\lambda}(x)=\sum_{w \in W_{0}} x^{\ell_{R}\left(t_{\lambda} w\right)}$ for $\lambda \in L$ has properties similar to $f_{0}(x)$, the Solomon polynomial discussed in Proposition 2.8. All we can say at the moment (as a consequence of Proposition 4.1) is that when $\lambda$ is $k$-dimensional, the polynomial $f_{\lambda}(x)$ has degree at most $k+n$ and is divisible by $t^{k}$.

\section{An unbounded example}

When $W$ is a Coxeter group that is neither finite nor affine, we conjecture the following.

Conjecture 5.1 (No upper bounds) The reflection length function on a Coxeter group $W$ has a uniform upper bound if and only if $W$ is of spherical or affine type.

We are not currently able to prove this conjecture, but we can show that it holds in at least one special case. Let $W$ be the free Coxeter group on three generators, i.e., the group generated by three involutions and with no other relations. Using a criterion of Dyer [3], we can show that the $n$th power of the product of the three standard generators has reflection length $n+2$. In particular, the reflection length function is unbounded on $W$, and Conjecture 5.1 holds in this case. We note that $W$ can be viewed as a reflection group acting on the hyperbolic plane generated by the reflections in the sides of an ideal triangle. Proving that all hyperbolic triangle Coxeter groups have unbounded reflection length would be a first step toward proving Conjecture 5.1.

Acknowledgements We would like to thank Matthew Dyer and John Stembridge for helpful early conversations about the ideas in this note, Rob Sulway for conversations about related topics that arise in his dissertation, and the anonymous referees for the helpful comments.

\section{References}

1. Bessis, D.: The dual braid monoid. Ann. Sci. Ecole Norm. Super. 36(5), 647-683 (2003)

2. Carter, R.W.: Conjugacy classes in the Weyl group. Compos. Math. 25, 1-59 (1972)

3. Dyer, M.J.: On minimal lengths of expressions of Coxeter group elements as products of reflections. Proc. Am. Math. Soc. 129(9), 2591-2595 (2001)

4. Humphreys, J.E.: Reflection Groups and Coxeter Groups. Cambridge Studies in Advanced Mathematics, vol. 29. Cambridge University Press, Cambridge (1990)

5. McCammond, J.: Noncrossing partitions in surprising locations. Am. Math. Mon. 113(7), 598-610 (2006)

6. Solomon, L.: Invariants of finite reflection groups. Nagoya Math. J. 22, 57-64 (1963) 